\title{
KONDO-LATTICE METALLIC AND SEMICONDUCTING STATES AND THEIR INSTABILITIES
}

\author{
J. SPALEK* \\ Marian Smoluchowski Institute of Physics, Jagiellonian University \\ Reymonta 4, 30-059 Kraków, Poland
}

We review briefly recent results concerning the stability of para-, ferro-, and antiferromagnetic states in the Kondo-lattice limit. Both the macroscopic quantities such as the specific heat and the electrical resistivity, as well as the magnitude of the magnetic moment and the mass enhancement (together with its spin dependence) are discussed.

PACS numbers: 71.27.+a, 71.10.-w, 71.10.Fd

\section{Introduction}

The heavy-electron Fermi liquid and the Kondo insulating state in the intermetallic compounds involving instability of the atomic $f$-electron shell ( $4 f$ in case of $\mathrm{Ce}$ or $\mathrm{Yb}$, and $5 f$ in case of $\mathrm{U}$ or Np elements), have been modelled with the help of periodic Anderson model (PAM) or its derivatives for paramagnetic [1], magnetic [2], and superconducting [3] states. This approach has also been applied to the paramagnetic Kondo insulators [4]. Such a simple model, particularly in the version when the hybridization matrix element $V_{k}$ is the wave-vector- $k$ independent (i.e. of the intraatomic character), cannot account for an anisotropy of physical properties such as magnetization. Nevertheless, it provides some of the basic features of the heavy-fermion state such as the extremely heavy effective masses, an almost integer occupancy $\left(n_{f} \approx 1\right)$ of the $f$ level, as well as obtained very recently [5] almost compensated moments in the antiferromagnetic metallic (spin-density-wave) state. The pairing due to the antiferromagnetic Kondo-type interactions [6] or mediated by the related spin fluctuations [7] has also been proposed, although the coexistence of antiferromagnetism and superconductivity has not been treated accurately from a microscopic point of view [8].

In this brief overview we concentrate on the paramagnetic and antiferromagnetic states at the threshold of their stability. In particular, we discuss the divergent behavior of the contributions due to quantum and spin fluctuations

*e-mail address: ufspalek@if.uj.edu.pl 
when approaching magnetic instability point [9], from the paramagnetic side, as well as summarize briefly the same point from the magnetic side [5]. Unfortunately, this type of approach starting from a stable Fermi-liquid (or insulating) state does not provide any clue into the observed fundamentally new phenomenon of the non-Fermi-liquid behavior [10], which requires a different type of approach from the outset (the incorporation of quantum critical fluctuations and/or of the atomic disorder).

\section{Paramagnetic state and its instability}

\subsection{Mean-field (saddle-point) solution}

As we mentioned above, the discussion of the heavy-fermion properties in the Kondo-lattice limit starts from the electronic model depicted schematically in Fig. 1 and represented by the Anderson-lattice Hamiltonian.

$$
\begin{aligned}
H= & \sum_{\boldsymbol{k}_{\sigma}} \varepsilon_{\boldsymbol{k}} c_{\boldsymbol{k}_{\sigma}}^{\dagger} c_{\boldsymbol{k}_{\sigma}}+\varepsilon_{f} \sum_{i \sigma} a_{i \sigma}^{\dagger} a_{i \sigma}+U \sum_{i} n_{i \uparrow} n_{i \downarrow} \\
& +\frac{V}{\sqrt{N}} \sum_{i k}\left(\mathrm{e}^{\mathrm{i} \boldsymbol{k} \cdot \boldsymbol{R}_{i}} c_{\boldsymbol{k}_{\sigma}}^{\dagger} a_{i \sigma}+\text { h.c. }\right)
\end{aligned}
$$

where $c_{k \sigma}^{\dagger}$ and $a_{i \sigma}^{\dagger}$ are the creation operators of the conduction (c) and localized $(f)$ electrons, with spin $\sigma$, respectively. The remaining symbols are standard [5]. The difficulty in solving this model is connected with the circumstance that the magnitude of $U \approx 5-6 \mathrm{eV}$ is usually the largest parameter in the system, since the bare band width of the conduction band is in the range $W \approx 1-2 \mathrm{eV}$, whereas the position of the bare atomic $f$-level is $\varepsilon_{f}=1-2 \mathrm{eV}$ below the Fermi level $\mu$, and the magnitude of hybridization is $|V|=0.1-0.5 \mathrm{eV}$ in a typical heavy-fermion material.

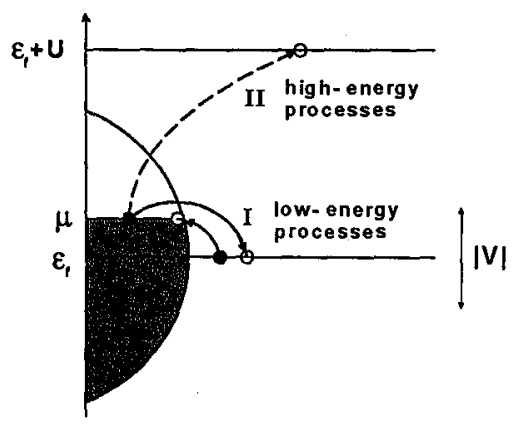

Fig. 1. The hybridization processes $(f-c$ mixing): high energy processes $\left(\sim V /\left(\varepsilon_{f}+U-\mu\right)\right)$ and the low energy mixing $\left(\sim V /\left(\varepsilon_{f}-\mu\right)\right)$. These processes combined together lead in the second order to the Kondo coupling in the Schrieffer-Wolff approximation. 
Various methods of approach have been used in solving this model in the lattice case [11]. They all converge to the mean-field (saddle-point) solution for the Fermi-liquid ground state, in which a new energy scale called the Kondo (or hybridization) temperature $T_{\mathrm{K}}$ emerges, where for finite but large $U$ we have that [9]

$$
k_{\mathrm{B}} T_{\mathrm{K}} \equiv \tilde{\varepsilon}_{f}-\mu=\frac{W}{2} \exp \left(-\frac{1}{\rho_{0} J}\right),
$$

where $\rho_{0}$ is the density of states in the bare band at the Fermi level, $J=$ $2 V^{2} /\left[\left|\varepsilon_{f}\right|\left(U-\left|\varepsilon_{f}\right|\right)\right]$ is the magnitude of the Kondo coupling between $c$ and $f$ electrons obtained by Schrieffer and Wolff and $\tilde{\varepsilon}_{f}$ is the position of the renormalized $f$-level. One should note that this result is derived also in the Kondo-lattice limit, i.e. for $\left(\mu-\varepsilon_{f}\right) \gg \rho_{0} V^{2}$. In that limit, the deviation from integer occupancy $n_{f}$ of the $f$-level is

$$
1-n_{f} \simeq\left(\frac{U-\left|\varepsilon_{f}\right|}{U}\right)^{2} \frac{k_{\mathrm{B}} T_{\mathrm{K}}}{2 \rho_{0} V^{2}} \sim \frac{W}{V^{2}} k_{\mathrm{B}} T_{\mathrm{K}} \ll 1 .
$$

Moreover, the density of hybridized quasiparticles at the Fermi level is

$$
\rho(E=\mu)=\frac{\tilde{V}^{2}}{W} \frac{1}{\left(\tilde{\varepsilon}_{f}-\mu\right)^{2}}=\frac{1}{2 k_{\mathrm{B}} T_{\mathrm{K}}}=\frac{1}{W}\left(\frac{W}{2 k_{\mathrm{B}} T_{\mathrm{K}}}\right) .
$$

Thus, since $W / k_{\mathrm{B}} T_{\mathrm{K}} \gg 1$, it is irrelevant what sort of bare density of states $\rho_{0}$ is taken. Hence, one can take it in the simplest form $\rho^{0}(\varepsilon)=1 / W$, for $-W / 2 \leq \varepsilon \leq$ $W / 2$, where $W$ is the bare band width. In such a situation, the gap between the hybridized (quasiparticle) bands is equal to

$$
\Delta_{\mathrm{G}}=2 k_{\mathrm{B}} T_{\mathrm{K}} \simeq \frac{\tilde{V}^{2}}{W},
$$

where $\tilde{V}$, as in formula (4), is the renormalized hybridization, which in the limit $U \rightarrow \infty$ takes the form $\tilde{V}=V\left[\left(1-n_{f}\right) /\left(1-n_{f} / 2\right)\right]^{1 / 2}$. Also, from Eq. (4) follows that $\rho / \rho_{0} \equiv m^{*} / m_{0}=W /\left(2 k_{\mathrm{B}} T_{\mathrm{K}}\right)$, where $m^{*} / m_{0}$ is the effective-mass enhancement due to the electronic correlations. One should note that since the density of quasiparticle states is $\sim 1 / T_{\mathrm{K}}$ (cf. Eq. (4)), and the low energy scale of electronic states is $\simeq T_{\mathrm{K}}$, then the total number of states in this interval is $\rho(\mu) \Delta E \approx 1$, i.e. almost all quasiparticles are located in the vicinity of the Fermi level. However, cautionary remark is in place here: this type of analysis is relevant if the number of particles per atom $n \leq 2$, i.e. when only the lowest quasiparticle band is filled (the upper hybridized band is empty and there is no electron-hole symmetry, since $\left.\varepsilon_{f}<0\right)$.

Physically, the results obtained in the saddle-point approximation mean that for the number of electrons per site $n<2$, the ground state is metallic and can be directly measured from the linear specific heat coefficient, which for a mole of compound takes the form $\gamma=\frac{\pi^{2}}{3} \frac{R}{T_{\mathrm{K}}}$, where $R$ is the gas constant. For example, for $\mathrm{CeRu}_{2} \mathrm{Si}_{2} \gamma=355 \mathrm{~mJ} /\left(\mathrm{mol} \mathrm{K}^{2}\right)$ and therefore, $T_{\mathrm{K}} \approx 80 \mathrm{~K}$. On the other hand, for the system for $n=2$ is a correlated band insulator called the Kondo insulator (a semiconductor at temperature $T>0$ ). 


\subsection{Effect of quantum fluctuations}

When we take into account the deviation from the saddle-point (mean-field) approximation the two corrections appear. First of them is due to the quantum Gaussian fluctuations of auxiliary (slave) boson fields. The second is due to the spin fluctuations. We characterize briefly the results for each of them (for details, see [9]).

The contribution to the specific heat (per site) due to the slave-boson fluctuations in the lowest order is

$$
\begin{aligned}
C_{V}^{\mathrm{SB}} & =\frac{1}{3} \pi^{2} k_{\mathrm{B}} \frac{T}{T_{\mathrm{K}}}\left(1+\frac{3}{2} \ln \left|1-\frac{q_{\mathrm{c}}^{2}}{\left|2 \kappa k_{\mathrm{F}}^{2}\right|}\right|\right) \\
& +\frac{\pi^{2}}{10 \kappa^{2}\left(v_{\mathrm{F}}^{*}\right)^{3}}\left(1-\frac{\pi^{2}}{12 \kappa}\right) k_{\mathrm{B}}^{4} T^{3} \ln \left(\frac{T}{T_{\mathrm{K}}}\right)+\beta_{\mathrm{SB}} T^{3},
\end{aligned}
$$

where the renormalized Fermi velocity is $v_{\mathrm{F}}^{*}=\hbar k_{\mathrm{F}} / m^{*}, \kappa \approx 1+o\left[\left(m_{0} / m^{*}\right)^{2}\right]$, and the last term is the higher order contribution. The parameter $q_{\mathrm{c}}$ is the cutoff frequency and as long as $q_{\mathrm{c}} \ll k_{\mathrm{F}}$, it does not influence much the results. They correspond to virtual charge excitations only because the Bose fields do not carry the spin.

The contribution due to the spin fluctuations is more involved. First, we have to derive effective spin-spin interactions and then calculate the dynamic susceptibility in the random phase approximation (RPA). In the system with ferromagnetic fluctuations (peaked around the wave vector $q \approx 0$ ) we obtain

$$
\begin{aligned}
C_{V}^{\mathrm{F}} & =\frac{3 \pi^{2}}{2} k_{\mathrm{B}}^{2} \frac{T}{T_{\mathrm{K}}} \ln \left|1-\frac{q_{\mathrm{c}}^{2}}{12 \alpha_{\mathrm{F}} k_{\mathrm{F}}^{2}}\right| \\
& +\frac{3 \pi^{3}}{10 \alpha_{\mathrm{F}}^{2}\left(v_{\mathrm{F}}^{*}\right)^{2}}\left(1+\frac{\pi^{2}}{12 \alpha_{\mathrm{F}}}\right) k_{\mathrm{B}}^{4} T^{3} \ln \left(\frac{T}{T_{\mathrm{K}}}\right)+\beta_{\mathrm{F}} T^{3},
\end{aligned}
$$

where

$$
\alpha_{\mathrm{F}}=\frac{1-I \rho(\mu)}{I \rho(\mu)}, \quad I=\frac{1}{4}\left[\frac{1}{\rho(\mu)}+\frac{1}{\rho_{0}} \ln ^{-2}\left(\frac{m^{*}}{m_{0}}\right)\right] .
$$

Note that $I$ plays the role of the effective local coupling, which is weaker for the systems with heavier masses. The contribution $C_{V}^{\mathrm{F}}$ diverges when the $\alpha_{\mathrm{F}} \rightarrow 0$, i.e. the system reaches the ferromagnetic instability. In the limit $\left(m^{*} / m_{0}\right) \rightarrow \infty$, we have that $I \rho(\mu) \rightarrow 1 / 4$, i.e. the system is paramagnetic even though the density of states at $\mu$ is very large, since the effective magnetic coupling is very weak.

A different type of behavior occurs when the mean-field susceptibility is peaked around the wave vector $Q=\left(Q_{1}, Q_{2}, Q_{3}\right)$. Around that point we can expand the susceptibility as [12]

Then

$$
\chi_{0}(q, \omega)=\chi_{0}(\boldsymbol{Q})\left[1-A \sum_{i=x, y, z}\left(q_{i}-Q_{i}\right)^{2}-B \omega^{2}+\mathrm{i} C \omega\right]
$$

$$
C_{V}^{\mathrm{AF}}=\frac{C q_{\mathrm{c}}}{6 \pi A}\left(1-\frac{\pi}{2 A^{1 / 2} q_{\mathrm{c}}} \alpha_{\mathrm{AF}}^{1 / 2}\right) k_{\mathrm{B}}^{2} T-\frac{\pi^{2} C^{3}}{120 A^{3 / 2} \alpha_{\mathrm{F}}^{3 / 2}} k_{\mathrm{B}}^{4} T^{3}
$$


where

$$
\alpha_{\mathrm{AF}}=\frac{1-I \chi_{0}(Q)}{I \chi_{0}(Q)}
$$

is the enhancement factor. Again, at the threshold of spin density wave (SDW) instability $I \chi_{0}(Q)=1$, both terms are divergent (note the absence of $T^{3} \ln T$ term in this case).

We now turn to the electrical resistivity. The resistivity is proportional to the imaginary part of the self-energy $\Sigma$ and has the Fermi-liquid form

$$
\tilde{\rho}(\omega, T) \sim \frac{2 m^{*}}{n e^{2}} \operatorname{Im} \Sigma\left(k_{\mathrm{F}}, \omega\right)
$$

where

$$
\operatorname{Im} \Sigma\left(k_{\mathrm{F}}, \omega\right) \approx K_{\alpha} \frac{m^{*}}{k_{\mathrm{F}}^{2}}\left[\omega^{2}+(\pi T)^{2}\right] .
$$

The contribution due to the slave-boson fluctuations is

$$
K_{\alpha=\mathrm{SB}} \equiv B \simeq \frac{\pi}{32} \frac{q_{\mathrm{c}}}{k_{\mathrm{F}}} \text {. }
$$

The corresponding contribution due to the spin fluctuations in almost ferromagnetic systems is

$$
K_{\alpha=\mathrm{F}} \approx \frac{\sqrt{3} \pi}{8} \alpha_{\mathrm{F}}^{-3 / 2}\left[\arctan \left(\frac{q_{\mathrm{c}}}{2 \sqrt{3 \alpha_{\mathrm{F}}}}\right)+\frac{q_{\mathrm{c}}}{2 \sqrt{3 \alpha_{\mathrm{F}}} k_{\mathrm{F}}}\right],
$$

whereas in almost antiferromagnetic systems

$$
K_{\alpha=\mathrm{AF}}=\frac{1}{A Q^{2}}\left(\frac{1}{\alpha_{\mathrm{AF}}}-\frac{1}{\alpha_{\mathrm{AF}}+A q_{\mathrm{c}}^{2}}\right) .
$$

Therefore, the total resistivity is

$$
\tilde{\rho}=\frac{2\left(B+K_{\alpha}\right)}{n e^{2} k_{\mathrm{F}}^{2}}\left(m^{*}\right)^{2}\left[\omega^{2}+(\pi T)^{2}\right] \sim \frac{\omega^{2}+(\pi T)^{2}}{T_{\mathrm{K}}^{2}} .
$$

One should note that close to the magnetic instability $\left(\alpha_{\mathrm{F}(\mathrm{AF})} \rightarrow 0\right) K_{\alpha}$ varies considerably, violating the simple Kadowaki-Woods scaling $\tilde{\rho}(\omega=0, T) \sim \gamma^{2} T^{2} \sim$ $\left(T / T_{\mathrm{K}}\right)^{2}$.

In general, the whole meticulously built Fermi-liquid picture breaks down at the magnetic quantum critical point $\alpha_{\mathrm{F}(\mathrm{AF})}=0$. However, the perturbative expansion presented above does not provide us with any clue about the possible non-Landau (non-Fermi liquid) type of behavior.

\section{Magnetic solutions: saddle-point approximation}

\subsection{Antiferromagnetic metallic phase}

The magnetic phases including the Kondo compensated phases have been recently analyzed in detail in Ref. [5] (for overview of the solution, see [13]). In this manner, a true Kondo lattice state was achieved for the number of electrons per site $n_{\mathrm{e}}=2-\delta$, with $\delta \ll 1$. The antiferromagnetic solution is stable for the perfectly nested case. This phase comprises the magnetic moment of $f$-electrons reduced by the circumstance that they hybridize and the whole system in the 


\section{Single-ion Kondo effect}

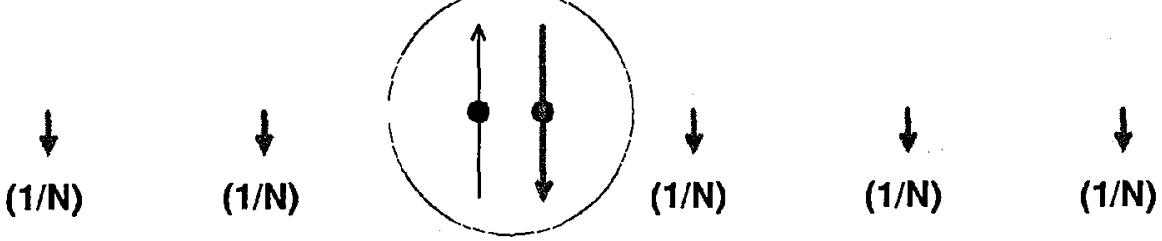

\section{Kondo lattice: Partial compensation}
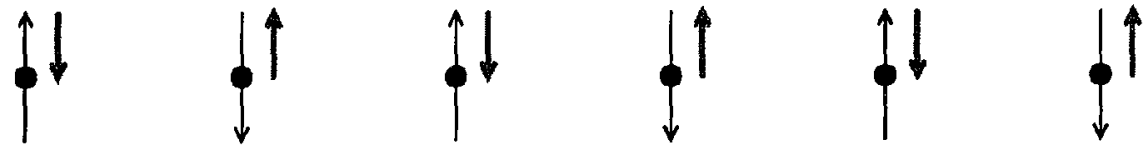

\section{Kondo lattice: Total compensation}
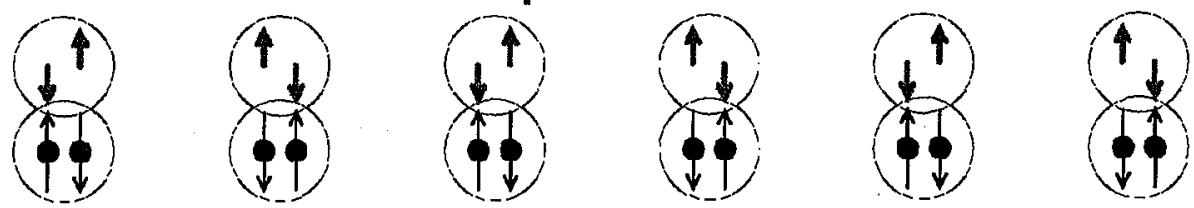

\section{Effectively}

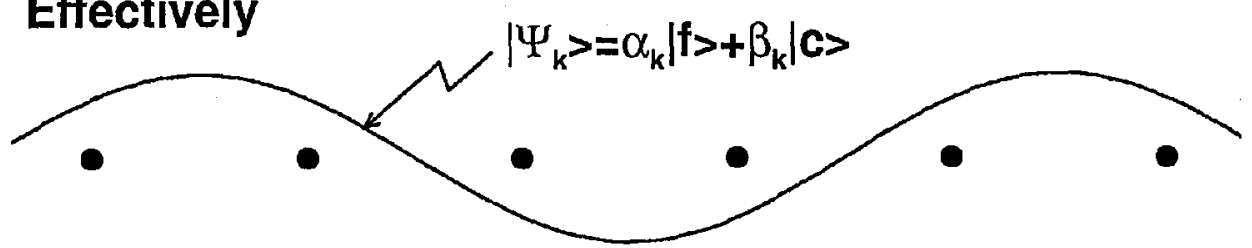

Fig. 2. Schematic representation of the total compensation of the $f$-moment in the impurity-Kondo case (top) and the partial compensation in the Kondo-lattice case (below). The totally compensated case in the lattice case corresponds to the simultaneous disappearance of the $c$ - and $f$-electron magnetic moments. The last case takes place for paramagnetic heavy fermions (bottom).

ground state is a Fermi liquid. Second, the conduction electrons are coupled to the electrons antiferromagnetically. However, only in the small-hybridization limit this coupling can be regarded as that of Schrieffer-Wolff type, i.e. $\sim V^{2}$. The effective situation is reproduced schematically in Fig. 2 , where we mark the $f$-moments as solid arrows and the opposite conduction-electron polarization as shaded vectors. One should note that while the carrier polarization (in the orbitally non-degenerate situation) compensates completely the localized $f$-moment in the impurity case, it compensates at most only a half of the atomic moment in the lattice case. The remaining part of the $f$-moment is the autocompensation which is complete for the hybridization $|V|$ exceeding a critical value. This is an intrinsic instability of the antiferromagnetic Kondo lattice in the ground state against the paramagnetic metallic state. At the instability point a weakly ferromagnetic phase (with the polarization $m=\delta$ ) may become a stable configuration. 
The results discussed above are not in complete agreement with those in the preceding section, where we have shown that the quantum fluctuation is stabilized if the effective (mean-field) mass is large (nota bene the last conclusion is in qualitative agreement with the observed experimental trend that the heaviest heavy-fermion systems such as $\mathrm{CeAl}_{3}, \mathrm{UBe}_{13}$, or $\mathrm{CeCu}_{6}$, which are paramagnetic). Obviously, to resolve the situation one would have to include the quantum fluctuations in analysis of magnetic phases, which represents a formidable task. It is also possible that the paramagnetic phase for e.g. Ce systems (containing one $f$-electron) may be additionally stabilized by the circumstance that we have more than one conduction electron per one $4 f$-electron (i.e. the overscreened situation in the Kondo-effect language). Such situation requires a separate analysis. For uranium systems such as $\mathrm{UPt}_{3}$ or $\mathrm{URu}_{2} \mathrm{Si}_{2}$ we have the situation with two $5 f$-electrons ( $5 f^{2}$ atomic configuration). In that case the Hund rule may drive the system towards magnetism. Obviously, such features are missing in the analysis performed in our papers $[5,13]$.

\subsection{Kondo insulating state}

The most of the above discussion can be applied equally well to the case with $n=2$, when the correlated (Kondo) insulating and magnetic state is formed. Such system is not a Mott-Hubbard insulator, since hybridization drives the system towards a band behavior. Indeed, a paramagnetic band insulating state is achieved for large enough hybridization [5, 13]. Probably, the most interesting feature, which one encounters in those semiconducting systems, is the fact that the gap disappears gradually with increasing temperature $T$ and vanishes at the temperature $T_{0}$ (we do not identify this temperature with $T_{\mathrm{K}}$, as $T_{\mathrm{K}}$ depends itself on $T$ ). Figure 3

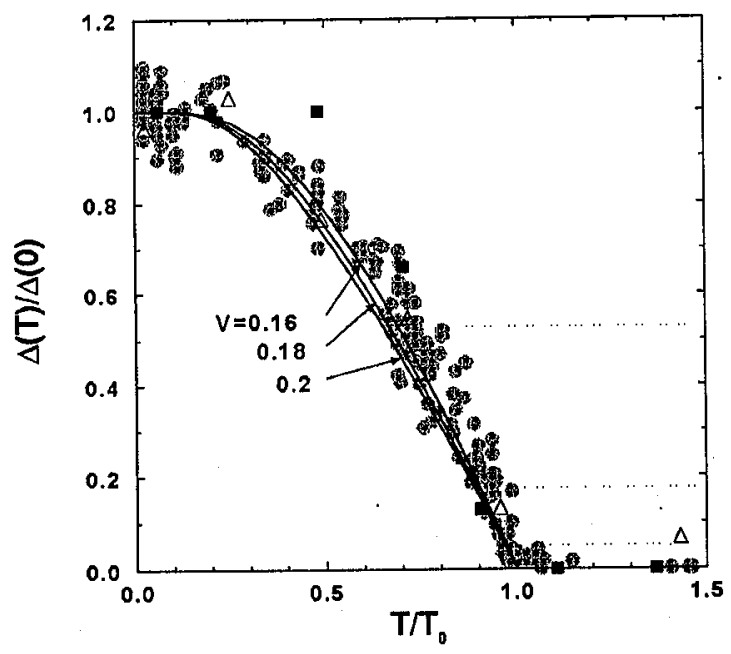

Fig. 3. Temperature dependence of the Kondo semiconducting gap: solid line - theory [16], circles - tunneling spectroscopy, squares - Raman spectroscopy, triangles infrared absorption (cf. papers grouped under Ref. [15]). 
displays the temperature dependence of the semiconducting gap measured for $\mathrm{FeSi}$ with the help of various techniques [14], whereas the solid lines represent the theoretical result for $\Delta_{G}(T)$ obtained independently $[15,5]$. One can say that the temperature $T_{0}$ represents an instability point of the paramagnetic Kondo semiconductor. Close to this point $\Delta_{G}(T) \sim\left(T_{0}-T\right)$. It is difficult to say if the temperature $T_{0}$ signals a phase transition (at least, in the paramagnetic phase).

\section{Conclusions}

For the three situations considered here: (i) paramagnetic Kondo-lattice metal with quantum fluctuations, (ii) heavy-fermion antiferromagnetic with almost compensated moments, and (iii) Kondo-lattice semiconductor with a vanishing gap, the starting phase becomes unstable. These systems are unstable because their microscopic parameters $\left(U / W, \varepsilon_{f} / W, V / W, n\right)$ are such that new energy scale arises characterized by $T_{\mathrm{K}}, I, T_{0}$, and the nonlinear molecular field $\beta_{3}$ not discussed here [5]. From the paramagnetic side this state becomes unstable if $k_{\mathrm{B}} T_{\mathrm{K}}<I$; this condition is similar to the Doniach criterion [16]. From magnetic point of view, the magnetism disappears when roughly the local molecular field $\beta_{3} \sim J$. A coherent picture of the magnetic instabilities from the two sides (paramagnetic and magnetic, respectively) is yet to emerge.

The microscopic understanding of heavy-fermion superconductivity remains as puzzling, as it was twenty years ago. We have also omitted the appearance of the spin-dependent masses in the applied field [17]; the occurrence of the spin-split masses provides a test of our mean-field approach starting from either Gutzwiller or slave-boson approach.

\section{Acknowledgment}

I would like to thank my two graduate students: Romek Doradziński (present) and Janek Karbowski (former), which have contributed remarkably with their meticulous analysis, to my understanding of the whole subject. It is unfortunate that the results reviewed briefly in Sec. 2.2 were never published due to the misunderstandings with a referee. The financial support of the Committee for Scientific Research through the grant No. 2P03B 12912 is also acknowledged.

\section{References}

[1] For early review see: D.M. Newns, N. Read, Adv. Phys. 36, 799 (1987); P.A. Lee, T.M. Rice, J.W. Serene, L.J. Sham, J.W. Wilkins, Comments Condens. Matter Phys. 12, 99 (1986).

[2] S. Doniach, Phys. Rev. B 35, 1814 (1987); F.J. Okhawa, Prog. Theor. Phys. Suppl. 108, 209 (1992); A.M. Reynolds, D.M. Edwards, A.C. Hewson, J. Phys. C 4, 7589 (1992); B. Möller, P. Wölfle, Phys. Rev. B 48, 10320 (1993).

[3] M. Lavagna, A.J. Millis, P.A. Lee, Phys. Rev. Lett. 58, 266 (1987); D.M. Newns, Phys. Rev. B 36, 2429 (1987); J. Spałek, Phys. Rev. B 38, 208 (1988); J. Karbowski, J. Spalek, Phys. Rev. B 49, 1454 (1994).

[4] P. Riseborough, Phys. Rev. B 45, 13984 (1992); V. Dorin, P. Schlottmann, Phys. Rev. B 46, 10800 (1992); P. Schlottmann, Phys. Rev. B 54, 12324 (1996); J. Karbowski, Phys. Rev. B 54, R728 (1996). 
[5] R. Doradziński, J. Spałek, Phys. Rev. B 56, R14239 (1997); Phys. Rev. B 58, 3293 (1998). For a complete analysis see R. Doradziński, Ph.D. thesis, Warsaw University, 1999.

[6] For review see: J. Spałek, J.M. Honig, in: Studies of High Temperature Superconductors, Ed. A. Narlikar, Vol. 8, Nova Science, New York 1991, p. 1.

[7] K. Miyake, S. Schmitt-Rink, C.M. Varma, Phys. Rev. B 34, 6554 (1986); M.R. Norman, Phys. Rev. Lett. 59, 232 (1987); Phys. Rev. B 37, 4987 (1988); Phys. Rev. B 41, 170 (1990).

[8] G.E. Volovik, L.P. Gor'kov, Pis'ma Zh. Eksp. Teor. Fiz. 39, 550 (1984) [JETP Lett. 39, 674 (1984)]; Zh. Eksp. Teor. Fiz. 88, 1412 (1985) [JETP 61, 843 (1985)]; M.J. Nass, K. Levin, G. Grest, Phys. Rev. Lett. 46, 614 (1981).

[9] J. Karbowski, Ph.D. thesis, Warsaw University, 1996; J. Karbowski, J. Spałek, preprint SPJU, Nos. 4/95 and 5/95, Jagiellonian University, Kraków 1995; the preprints are available on request from the author.

[10] For review see: J. Phys., Condens. Matter 8, No. 48 (1996).

[11] For a comparative study of various mean-field approaches see: F. Gebhard, Phys. Rev. $B$ 41, 992 (1991).

[12] T. Moriya, Phys. Rev. Lett. 24, 1433 (1970); H. Hasegawa, T. Moriya, J. Phys. Soc. Jpn. 36, 1542 (1974).

[13] J. Spałek, R. Doradziński, in: Magnetism and Electronic Correlations in Local-Moment Systems: Rare-Earth Elements and Compounds, Eds. M. D,onath, P.A. Dowben, W. Nolting, World Scientific, Singapore 1998, p. 387; Acta Phys. Pol. A 96, 677 (1999).

[14] M. Fäth, J. Aarts, A.A. Menovsky, G.J. Nieuwenhuys, J.A. Mydosh, Phys. Rev. B 58, 15483 (1998); P. Nyhus, S.L. Cooper, Z. Fisk, Phys. Rev. B 51, 15626 (1995); A. Damascelli, K. Schulte, D. van der Marel, A.A. Menovsky, Phys. Rev. B 55, R4863 (1997).

[15] R. Doradziński, J. Spałek, to be submitted.

[16] S. Doniach, Physica B 91, 231 (1977); B. Coqblin, J. Arispe, J.R. Iglesias, C. Lacroix, K. Le Hur, J. Phys. Soc. Jpn. 65, Suppl. B, 64 (1996).

[17] J. Spałek, R. Citro, Z. Phys. B 103, 267 (1997); R. Citro, J. Spałek, Physica B 230-232, 469 (1997). 